\title{
Attribute Based Image Duplication Alert Message Using Big Data
}

\author{
T.Sumallika ${ }^{1}$, Ch.Suguna Latha ${ }^{2}$, V. Alekya ${ }^{3}$, D. Hemasindhu ${ }^{4}$ \\ ${ }^{1}$ (Assisstant professor, Dept of Information Technology, Gudlavalleru Engineering College, Gudlavalleru. \\ India. \\ ${ }^{2}$ (Assisstant professor, Dept of Information Technology, Gudlavalleru Engineering College, Gudlavalleru. \\ India. \\ ${ }^{3}$ (Assisstant professor, Dept of Information Technology, Gudlavalleru Engineering College, \\ Gudlavalleru. India. \\ ${ }^{4}$ (Assisstant professor, Dept of Information Technology, Gudlavalleru Engineering College, Gudlavalleru. \\ India.
}

\begin{abstract}
Using this paper "Attribute Based Image Duplication Alert Message Using Big Data" the stranger cannot copy any of the photo which is related to the user and, we also provide fast image search. That means, the image will be searched by using the attribute name and the hyper graph learning method. The user will have a global vision on searching the photos. It will be visible and more easily findable. Users can search more within a short period. It mainly provides users privacy. We proposed a new attribute-assisted method based on hyper graph learning. First we have to train several classifiers for all the pre-defined. Attributes and each image is represented by attribute feature consisting of the responses from these classifiers. Semantic attributes are expected to narrow down the semantic gap between low-level visual features and high level semantic meanings. If any naïve user is trying to copy the images, Automatic message can be send to the users using Rabbit MQ when any stranger copy the image.
\end{abstract}

Keywords: attribute name, hyper graph learning method, automatic message, Rabbit MQ.

\section{INTRODUCTION}

Online Social Networks (OSN's) have become integral part of our daily life and has profoundly changed the way we interact with each other, fulfilling our social needs, the needs for social interactions, information sharing, appreciation and respect. The nature of social media makes people put more content, including photos, over OSNs without too much thought on the content. However, once something, (such as photo) is posted online, it becomes a permanent record, which may be used for many purposes we never expect. So there is a need to control the photo copying activity. With the dramatic increase of online images, image retrieval has attracted significant attention in both academia and industry. Many image search engines such as Google and Bing have relied on corresponding textual information of the images against queries given by users. However, text based image retrieval suffers from essential difficulties that are caused mainly by the inability of the related text to suitably describe the image Content. newly, visual re-ranking has been proposed to refine text-based search results by exploiting the visual information contained in the images. The existing visual re-ranking methods can be typically categorized into three categories as the clustering based, classification based and graph based methods. In any means the user must satisfy by the searching activity and he need to get appropriate results. A $\mathrm{z}$

\subsection{Social Achievability:}

The aspect of study is to check the level of acceptance of the system by the user. This includes the process of training the user to use the system efficiently. The user must not feel in danger by the system, instead must accept it as a necessity. The level of acceptance by the users solely depends on the methods that are employed to educate the user about the system and to formu late him known with it. His level of confidence must be raised so that he is also able to make some constructive criticis $\mathrm{m}$, which is welcomed, as he is the final user of the system.

\section{RELATED WORK}

To improve the performance of searching images visual search reranking is very good option[1]. In this section, existing visual search reranking approaches are explained along with semantic attributes and hyper graph learning[3]. To improve the accuracy of the text-based image search ranking, visual reranking has been proposed to improve the search result from the text-based image search engine by incorporating the data conveyed by the visual modality [3]. 


\subsection{Admin}

Admin can have all the rights. He can view the images, which were posted by the users.

Duties of Admin:

- List of users

- List of images with comments

- View search History

- View image ranks

- Search posts

\subsection{User}

User can access the photos from the website. He can post the photo, interact with friends, and also

Duties of the user:

- Profile

- Add post

- Search post based on keyword

- Search post based on content

\subsection{Image search}

This is a small database which consists of photo searching based on our require ment.

1) Search history: This is controlled by admin; the admin can view the search history details. If he clicks on search history button, it will give the list of searched user details with their tags such as user name, image name, time and date.

2) Rank of images: In user's section, the admin can view the list of ranking images. If admin selects list of ranking images, then the server will give list of images with their tags, and rank of image.

3) Upload images: The admin can upload n number of images. Admin want to upload new image then he has to enter some fields like image name, image color, image description, image type, image usage, browse the image file and upload. After uploading we get a response from the server. at first new uploaded image rank is zero. After screening that image rank will re-rank.

There may present $n$ number of users. All should register before the processing operation. And register user details are stored in user section. After registration user has to login by using authorized user name and password. Once the logged in will do some operations like checking details, searching images, request secrete key and logout. The user select details button then the server will all details such as user name, phone no, address, e mail ID and location. Before searching any images user should request a secrete key to admin, then the admin will produce a secrete key for specific user and send it to the user. After receiving a secrete key user can search the images base on query and fields like image name, image color, image usage and image type. And server will give details to the user, then that image rank will be increased. And it is difficult to the naive user to copy the images belongs to the user, and use it in different ways. If any naïve user is trying to copy the images, Automatic message can be send to the users using big data RabbitMQ, when any stranger tries to copy the image.

The important idea behind this messaging model in RabbitMQ is the producer can send messages to an exchange and An exchange on one side it receives messages from producers and the other side it will push them to queues. (Fig: 1)The exchange must know exactly what to do with a message it receives.

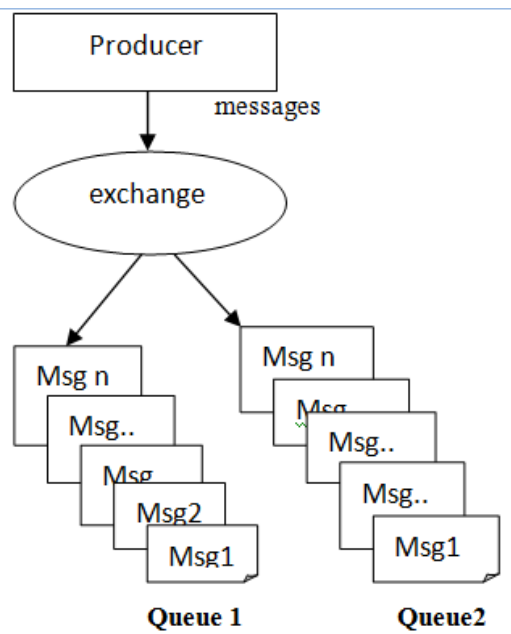

Fig 1: message delivery process in RabbitMQ

There are different types of exchanges available: direct, topic, headers and fanout.

We focused on the last one -- the fanout.

channel.exchange_declare(exchange='logs',type='fano ut')

The fanout exchange broadcasts all the received messages to all the queues it is connected.

RabbitMQ is a message agent. The primary idea is very simple: it accepts and forwards messages. This is like a post office: if we send mail to the post box Postman will deliver the mail to the receiver. Using this metaphor RabbitMQ is a post box, a post office and a postman.

The major difference between RabbitMQ and the post office is the fact that it doesn't deal with paper, instead it accepts, stores and forwards binary lump of data - messages.

We will stock up the addresses of images and accessing points of images in HDFS, if any naïve user accessed any image in the HDFS the fanout exchange will identify the image address and the IPaddress which the naïve user accessed and sends detailed message to the image address so that we can know the agents who are using the stuff in a negative way as shown in the fig 2 . 


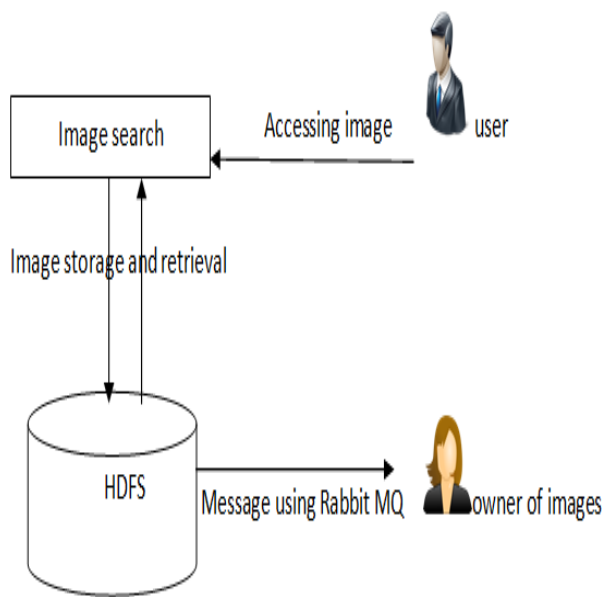

Fig 2: illustration of proposed system

\section{CONCLUSION}

Control of photo copying and Quick image search is a web based application designed to avoid photo copying and easy search. It also provides user security. By using this user can search images quickly. The searching can be done in two ways by attribute and text based search. The user can search an image by entering the attribute name. This provides searching activity based on image colour and type. If any naïve user is trying to copy the images, Automatic message can be send to the users using RabbitMQ when any stranger copy the image. the stranger cannot copy any of the photo which is related to the user and, we also provide fast image search

\section{REFERENCES}

Journal Papers:

[1]. Snehal S Patil , A jay Dani, "Attribute Based Image Search Re-Ranking" in IJSR Volume 4 Is sue 11, Nove mber 2015

[2]. IEEE TRANSACTIONS ON IMAGE PROCESSING VOL. 24, NO. 1, JANUARY 2015 IEEE paper: An Attribute-Assisted Reran king Model for Web Image Search. Junjie Cai, Zheng-Jun Zha, Member, IEEE, Meng Wang, Shiliang Zhang, and Qi Tian, Senior Member, IEEE

[3]. IEEE TRANSACTIONS ON MULTIMED VOL.14, NO.3, JUNE 2012IEEE paper: Prototype-Based Image Search Reranking. Linjun Yang, Member, IEEE, and Alan Hanja lic, Sen ior Me mber, IEEE.

[4]. S. Changpinyo and E. Sudderth. Learning image attributes using the Indian Buffet Process. Technical report, Brown University, 2012.

[5]. B. Siddiquie, R. S. Feris, and L. S. Davis, "Image ranking and retrieval based on multiattribute queries," in Proc. IEEE Conf. Comput. Vis. Pattern Recognit., Jun. 2011
[6]. Learning Scalable Discriminative Dictionary with Sample Relatedness. Jiashi Feng1, Stefanie Jegelka, Shuicheng Yan, Trevor Darrell Department of ECE, National University of Singapore, Singapore Department of EECS \& ICSI, UC Berkeley, USA

[7]. N. Ku mar, A. C. Berg, P. N. Belhumeur, and S. K. Nayar, "Attribute and simile classifiers for face verification," in Proc. IEEE Int. Conf. Comput. Vis., Sep./Oct. 2009, pp. 365-372

[8]. Attribute Based Image Classification and Retrieval Using visual attributes to search for fashion items. Master's Thesis

Urls:

[1]. Net beans downloads:www.filehippo.com/download_netb eans

[2]. Java books :bookboon.com/en/javaprogramming-language-ebooks

[3]. Jsp tages: www.tutorialspoint.com/jsp/jsp_standard_tag_1 ibrary.htm

[4]. HTML codes:www.w3schools.com/html/html_exampl es.asp

[5]. www.tutorials point.com/jdbc/jdbc-dbconnections.htm

[6]. ttps://www.rabbit mq.co m/tutorials/tutorial-onepython.html

Books:

[1]. Database and System Design by Korth

[2]. Software Eng ineering Concepts by Robert Pressman 